GUEST EDITORIAL

\title{
Perioperative evaluation of patients who are due to undergo surgery
}

Physicians are often requested to evaluate patients before surgery, either in response to a request from a surgeon or a primary care clinician assessing the patient prior to surgical referral. The objectives of this preoperative evaluation are to determine the risk to the patient of the proposed procedure and to minimise risk by: (i) identifying unrecognised comorbid disease and risk factors for medical complications of surgery; (ii) optimising the preoperative medical condition; (iii) recognising and treating potential complications; and (iv) working effectively as a member of the preoperative team (including those from nursing, medical, surgical and anaesthetic backgrounds).

Does preoperative evaluation of patients improve surgical outcomes? Data from published studies reveal that physicians and anaesthetists are more likely to identify conditions that may affect surgical outcomes. They then recommend interventions for these condition $^{[1,2]}$ and occasionally cancel or delay surgery so that medical conditions can be optimally managed, ${ }^{[3]}$ ensuring a high level of satisfaction with co-management arrangements. ${ }^{[4]}$

Data on the effect of preoperative medical consultation on cost measures are conflicting. Three large studies reported a decrease in hospital stay after perioperative evaluation and care of patients undergoing thoracic, ${ }^{[5]}$ hip $^{[6]}$ and various other operations. ${ }^{[7]}$ Some studies, however, showed increased costs and a similar length of stay for patients who had been consulted. ${ }^{[4,8]}$

Similarly, studies of the impact of perioperative medical evaluation on perioperative mortality are contradictory. In an investigation of neurosurgical patients, medical consultation had no effect on mortality, ${ }^{[4]}$ while in a different study, it was shown to increase 30-day and 1-year mortality rates and length of hospital stay, respectively. ${ }^{[9]}$

Overall, robust evidence demonstrating clear improvements in resource utilisation or patient outcomes is currently lacking. Nevertheless, the practice of perioperative evaluation is widespread and, assuming doctors make evidence-based recommendations that improve surgical outcomes, it is reasonable to infer that consultation will improve the care of the surgical patient if consultative recommendations are implemented.

Closer to home, perioperative research remains unco-ordinated in South Africa (SA). A group of investigators and interested individuals collaborated under the auspices of the SA Perioperative Research Group (SAPORG). Members of SAPORG believe that: (i) collaborative research is necessary to address the clinical challenges encountered in perioperative care and outcomes, both in SA and globally; (ii) there is capacity to conduct national and international collaborative research in SA; (iii) collaborative research conserves limited research resources; (iv) there are urgent public health issues in perioperative medicine that need to be addressed to improve the health of the SA population; and $(v)$ a national research prioritysetting process is necessary to prioritise research in an environment of limited research resources. ${ }^{[10]}$

To this end, SAPORG has defined 10 research priorities for perioperative medicine in SA: (i) the establishment of a national database of critical care outcomes and critical care resources; (ii) a randomised controlled trial of preoperative B-type natriuretic peptide-guided medical therapy to decrease major adverse cardiac events after noncardiac surgery; (iii) a national prospective observational study of the outcomes associated with paediatric surgical cases; (iv) a national observational study of maternal and fetal outcomes following operative delivery in SA; $(v)$ a stepped-wedge trial of an enhanced recovery-after-surgery programme; $(\mathrm{vi})$ a stepped-wedge trial of a surgical safety checklist of patient outcomes in SA; (vii) a prospective observational study of perioperative outcomes after surgery in district general hospitals in SA; (viii) short-course interventions to improve anaesthetic skills of rural doctors; (ix) studies of the efficacy of simulation training to improve patient outcomes, team dynamics and leadership; and $(x)$ the development and validation of a risk stratification tool for SA surgery based on the SA Surgical Outcomes Study (SASOS) data. ${ }^{[10]}$

A recent publication in the Lancet reported on a 7-day, international, prospective, observational cohort study of patients aged $\geq 18$ years undergoing any inpatient surgery in 25 countries in Africa (African Surgical Outcomes Study). ${ }^{[11]}$ A total of 11422 patients were included from 247 hospitals serving a median population of 810 000, with a combined number of specialist surgeons, obstetricians and anaesthetists of $0.7 / 100000$ population. A median of 212 surgical procedures per 100000 population were performed in hospitals each year. Patients were younger (mean age 38.5 years) and had a lower risk profile than that reported in high-income countries. Patients (11\%) were infected with HIV, 57\% of procedures were urgent or emergent, and the most common procedure was caesarean delivery (33\%). Postoperative complications occurred in $18.2 \%$, and $2.1 \%$ of patients died the day after surgery. In this important publication, despite a low-risk profile and few postoperative complications, patients in Africa were twice as likely to die after surgery compared with the global average for postoperative deaths. The authors conclude that initiatives to increase access to surgical treatments in Africa therefore should be coupled with improved surveillance for deteriorating physiology in patients who develop postoperative complications and the resources necessary to achieve this objective. ${ }^{[11]}$

In this CME issue, $\mathrm{Du}$ Toit et al. ${ }^{[12]}$ review the perioperative management of diabetes. In their comprehensive summary, the authors review the guidelines for optimisation and perioperative management of diabetic patients, and importantly place their discussion within the SA context. Ultimately, perioperative diabetic care should be driven by a multidisciplinary team considering the evidence base within a resource and patient context.

The second article, by Neethling et al., ${ }^{[13]}$ discusses the role of point-of-care ultrasound (POCUS) as an essential modality in the assessment of critically ill patients and those in the perioperative period. POCUS can be performed by trained non-cardiologist physicians at the patient's bedside as an adjunct to the physical examination, and aids with the rapid diagnosis of severe and life-threatening pathological conditions, often changes clinical management and may have an impact on patient outcomes.

While no large studies have definitively shown a decrease in perioperative morbidity associated with perioperative medical consultation, the practice is nevertheless widespread and, assuming that consultants make evidence-based recommendations that improve surgical outcomes, it is reasonable to infer that consultation will improve the care of the surgical patient. The experienced 
perioperative medicine physician should be able to identify the pertinent medical problems, anticipate potential perioperative problems, avoid addressing issues outside of their area of expertise or issues unrelated to the procedure, assess a patient's risk and need for further interventions, and communicate effectively with the surgeon and anaesthesiologist. There is now emerging evidence of the status of perioperative medicine and outcomes on the African continent. In the future, it is my hope that further research in this area will improve surgical outcomes of patients in SA and beyond.

\section{Conflicts of interest. None.}

Funding. This manuscript is not funded. Prof. N Ntusi gratefully acknowledges support from the National Research Foundation and the Medical Research Council of South Africa, as well as the Harry Crossley Foundation.

\section{Ntobeko Ntusi}

Division of Cardiology, Department of Medicine, Faculty of Health Sciences, University of Cape Town and Groote Schuur Hospital; Cape Universities Body Imaging Centre, Faculty of Health Sciences, University of Cape Town; and Hatter Institute of Cardiovascular Research in Africa, Department of Medicine, Faculty of Health Sciences, University of Cape Town, South Africa ntobeko.ntusi@uct.ac.za

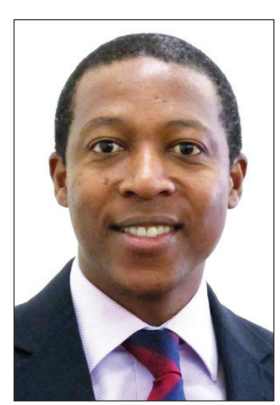

1. Devereaux PJ, Ghali WA, Gibson NE, et al. Physicians' recommendations for patients who undergo noncardiac surgery. Clin Invest Med 2000;23(2):116-123.

2. Clelland C, Worland RL, Jessup DE, East D. Preoperative medical evaluation in patients having joint replacement surgery: Added benefits. South Med J 1996;89(10):958-960. https://doi. joint replacement surgery: Added ben

3. Mollema R, Berger P, Girbes AR. The value of peri-operative consultation on a general surgical ward by the internist. Neth J Med 2000;56(1):7-11. https://doi.org/10.1016/s0300-2977(99)00081-9 4. Auerbach AD, Wachter RM, Cheng HQ, et al. Comanagement of surgical patients between neurosurgeons and hospitalists. Arch Intern Med 2010;170(22):2004-2010. https://doi.org/10.1001/ archinternmed.2010.432

5. Macpherson DS, Parenti C, Nee J, Petzel RA, Ward H. An internist joins the surgery service: Does comanagement make a difference? J Gen Intern Med 1994;9(8):440-444. https://doi.org/10.1007/ bfo2599059

6. Phy MP, Vanness DJ, Melton LJ, et al. Effects of a hospitalist model on elderly patients with hip 6. Phy MP, Vanness DJ, Melton LJ, et al. Effects of a hospitalist model on elderly patients w
fracture. Arch Intern Med 2005;165(7):796-801. https://doi.org/10.1001/archinte.165.7.796

fracture. Arch Intern Med 2005; 165(7):796-801. https://doi.org/10.1001/archinte.165.7.796
7. Vazirani S, Lankarani-Fard A, Liang LJ, Stelzner M, Asch SM. Perioperative processes and outcomes after implementation of a hospitalist-run preoperative clinic. J Hosp Med 2012;7(9):697-701. https:// doi.org/10.1002/jhm. 1968

8. Auerbach AD, Rasic MA, Sehgal N, Ide B, Stone B, Maselli J. Opportunity missed: Medical consultation, resource use, and quality of care of patients undergoing major surgery. Arch Intern Med 2007;167(21):2338-2344. https://doi.org/10.1001/archinte.167.21.2338

9. Wijeysundera DN, Austin PC, Beattie WS, Hux JE, Laupacis A. Outcomes and processes of care related to preoperative medical consultation. Arch Intern Med 2010;170(15):1365-1374. https://doi. org/10.1001/archinternmed.2010.204

10. Biccard BM, Alphonsus CS, Bishop DG, et al. National priorities for perioperative research in South Africa. S Afr Med J 2016;106(5):58-59. https://doi.org/10.7196/SAMJ.2016.v106i5.10269

11. Biccard BM, Madiba TE, Kluyts HL, et al; African Surgical Outcomes Study (ASOS) investigators. Perioperative patient outcomes in the African Surgical Outcomes Study: A 7-day prospective observational cohort study. Lancet 2018;(epub ahead of print). https://doi.org/10.1016/S01406736(18)30001-1

12. Du Toit L, Biesman-Simons T, Levy N, Dave JA. A practical approach to managing diabetes in the perioperative period. S Afr Med J 2018;108(5):369-375. https://doi.org/10.7196/SAMJ.2018.v108i5. 13311

13. Neethling E, Roodt F, Beck C, Swanevelder JLC. Point-of-care and lung ultrasound incorporated in daily practice. S Afr Med J 2018;108(5):376-381. https://doi.org/10.7196/SAMJ.2018.v108i5.13313

S Afr Med J 2018;108(5):367-368. DOI:10.7196/SAMJ.2018.v108i5.13329 